\title{
Filtering for Discrete Fuzzy Stochastic Time-Delay Systems with Sensor Saturation
}

\author{
Jia You, ${ }^{1}$ Shen Yin, ${ }^{2}$ and Hamid R. Karimi ${ }^{3}$ \\ ${ }^{1}$ Research Institute of Intelligent Control and Systems, Harbin Institute of Technology, Heilongjiang 150001, China \\ ${ }^{2}$ College of Information Science and Technology, Bohai University, Jinzhou, Liaoning 121013, China \\ ${ }^{3}$ Department of Engineering, Faculty of Engineering and Science, University of Agder, 4879 Grimstad, Norway
}

Correspondence should be addressed to Shen Yin; shen.yin2011@googlemail.com

Received 29 November 2012; Accepted 4 February 2013

Academic Editor: Peng Shi

Copyright (C) 2013 Jia You et al. This is an open access article distributed under the Creative Commons Attribution License, which permits unrestricted use, distribution, and reproduction in any medium, provided the original work is properly cited.

\begin{abstract}
This paper addresses the $\mathscr{H}_{\infty}$ filtering problem for discrete fuzzy stochastic systems with time-varying delay and sensor saturation. Random noise depending on state and external disturbance is also taken into account. A decomposition approach is employed to solve the characteristic of sensor saturation. The scaled small gain (SSG) theorem is extended to the stochastic systems, which is employed to handle with the time-varying delay by transforming the original system into the form of an interconnected system consisting of two subsystems. By the proposed Lyapunov-Krasovskii function, the scaled small gains of the subsystems are analyzed, respectively. Sufficient conditions for the stochastic stability of the filtering error system with a prescribed $\mathscr{H}_{\infty}$ level are established such that the gains of the $\mathscr{H}_{\infty}$ filter can be obtained explicitly. Finally, simulation results are presented to demonstrate the effectiveness of the proposed approach.
\end{abstract}

\section{Introduction}

In recent years, the control and filtering problems for TakagiSugeno (T-S) fuzzy systems, which have been employed to solve a great deal of issues in control and filtering fields, have drawn a great attention of many researchers [1-4]. Described by a set of If-Then rules, the T-S fuzzy system can approximate any given continuous function in a compact set of $\mathscr{R}^{n}$ at any preciseness [5-7]. Consequently, it has become one of the most useful and popular research platforms in fuzzy logic control (FLC), since many nonlinear systems can be analyzed by using the properties of conventional linear systems via T-S fuzzy model. A great number of significant results have been reported in the literatures [8-11]. In particular, a great effort has been devoted to the filtering problems for T-S fuzzy systems. Among the existing filtering approaches, the Kalman filtering is popular and useful due to its easy implementing and good performance in many engineering problems [12]. However, the Kalman filtering has strict constraints that a precise model of the system is available and all error terms and measurements have Gaussian distributions, which are hard to reach in many practical dynamics. Meanwhile, $\mathscr{H}_{\infty}$ filtering, which only requires that the noise sources to be arbitrary signals with bounded energy or bounded average power instead of Gaussian noises, has arguably become another useful and important filtering method for T-S fuzzy systems.

On the other hand, a large number of practical engineering problems encounter time-delay phenomenon, which is always a source of poor performance and even instability of the systems $[13,14]$. Over the past few years, a great interest has been devoted to time-delay systems, and many significant results have been reported by the Lyapunov method [3, 15-17]. On the other hand, an input-output (IO) approach developed from the SSG theorem that has been introduced for constant delay $[18,19]$ and then extended to time-varying delay [20]. In contrast to the time-independent methods [15], less conservative results could be achieved by this approach, which analyzes the stability of the system by transforming the system into the form of two interconnected subsystems [20, 21], especially for small time delays [22]. It should be noted that a proper approximation to the time delay plays the key role in this method, and many approximation approaches are proposed to pursue smaller approximation error. Recently, 
a method for estimating the time-varying delay by the upper and lower delay bounds was introduced in [23], which has a much smaller resulting error than the other one-term ones [24-26]. This motivates this research to extend this approach to $\mathscr{H}_{\infty}$ filtering problems for T-S fuzzy systems.

In practical engineering, sensor saturation is one of the most popular phenomena that limits the performance of sensors on various aspects significantly. When sensors cannot provide the enough amplitude signals due to the physical or safety constraints $[18,27]$, a complicated controller is needed for a good resulting performance. In recent years, researchers have paid a great attention to filtering problems with sensor saturation [28, 29]. However, it should be noted that in [28] the output of the system is only the sum of a linear term and a nonlinear one, which means that the output model is a special class of nonlinear sensor model, rather than a general form, and the filtering method in [28] cannot be utilized for the stochastic systems directly [30, 31].

Motivated by the above reasons, we investigate the $\mathscr{H}_{\infty}$ filtering problem for discrete-time T-S fuzzy stochastic systems with time-varying delay and senor saturation. A decomposition approach is applied to handle with the characteristic of the sensor saturation, and random noise subject to state and external-disturbance is also considered. A new model transformation is employed to transform the original system into a form of two interconnected subsystems. Based on the SSG theorem developed to stochastic systems and the proposed Lyapunov-Krasovskii function, the SSG of each subsystem is analyzed to establish the sufficient conditions under which the filtering error system is stochastically stable with a prescribed $\mathscr{H}_{\infty}$ performance level $\gamma$. Then, the corresponding $\mathscr{H}_{\infty}$ filter design technique is proposed. Finally, an illustrative example is presented to show the effectiveness of the proposed method.

The remainder of this paper is organized as follows. In Section 2, the problem of $\mathscr{H}_{\infty}$ filtering is formulated for T-S fuzzy stochastic systems with time-varying delay and sensor saturation. Section 3 presents the results for $\mathscr{H}_{\infty}$ performance analysis and $\mathscr{H}_{\infty}$ filter design. A numerical example is provided to demonstrate the effectiveness of the designed filtering technique in Section 4 , and we conclude the paper with Section 5.

Notation. Throughout this paper, $\mathbb{R}^{n}$ represents the $n$ dimensional Euclidean space, $\mathbb{R}^{n \times m}$ is the set of all $n \times m$ real matrices, and the superscripts " -1 " and " $T$ ", respectively, stand for the matrix inverse and matrix transpose. $\operatorname{Sym}\{A\}$ is the shortened notation for $A+A^{T}$, and the notation $P>0$ (resp., $P \geq 0$ ), for $P \in \mathbb{R}^{n \times n}$, means that $P$ is real symmetric and positive definite (resp., semidefinite). The symmetric elements of the symmetric matrix are represented by an asterisk (*), and the block diagonal matrices are denoted by $\operatorname{diag}\{\cdots\} . \mathbf{G}_{1} \circ \mathbf{G}_{2}$ means the series connection of mapping $\mathbf{G}_{1}$ and $\mathbf{G}_{2} \cdot \mathbf{E}\{\cdot\}$ denotes the expectation operator with respect to probability measure, and for vector $x(k),\|x\|_{E_{2}}=$ $\mathbf{E}\left\{\sum_{n=0}^{\infty}\|x(n)\|^{2}\right\}^{1 / 2}$.

\section{Problem Formulation}

We consider the following nonlinear fuzzy dynamic described by a Takagi-Sugeno (T-S) stochastic model: Then

$R^{i}$ : If $\zeta_{1}(k)$ is $F_{1}^{i}, \zeta_{2}(k)$ is $F_{2}^{i}$ and $\ldots$, and $\zeta_{s}(k)$ is $F_{s}^{i}$,

$$
\begin{gathered}
x(k+1)=A_{i} x(k)+A_{d i} x\left(k-\tau_{k}\right)+B_{i} v(k) \\
+\left[E_{i} x(k)+G_{i} v(k)\right] w(k), \\
y(k)=\phi\left(C_{i} x(k)\right)+D_{i} v(k), \\
z(k)=L_{i} x(k),
\end{gathered}
$$

where $R^{i}, i \in\{1,2, \ldots, \bar{n}\}$, denotes the $i$ th fuzzy inference rule, $\bar{n}$ is the number of the inference rules, $F_{j}^{i}, j \in\{1,2, \ldots, s\}$, are fuzzy sets, and $\zeta(k)=\left[\zeta_{1}(k), \zeta_{2}(k), \ldots, \zeta_{s}(k)\right]$ are some measurable premise variables of the system. $x(k) \in \mathbb{R}^{n}$ is the state vector, $y(k) \in \mathbb{R}^{m}$ is the measured output, and $z(k) \in$ $\mathbb{R}^{p}$ is the signal to be estimated. $A_{i} \in \mathbb{R}^{n \times n}, A_{d i} \in \mathbb{R}^{n \times n}$, $B_{i} \in \mathbb{R}^{n \times q}, C_{i} \in \mathbb{R}^{m \times n}, D_{i} \in \mathbb{R}^{m \times q}, L_{i} \in \mathbb{R}^{p \times n}, E_{i} \in \mathbb{R}^{n \times n}$, and $G_{i} \in \mathbb{R}^{n \times q}$ are known constant matrices. $\tau_{k}$ is the timevarying delay satisfying

$$
d_{1} \leq \tau_{k} \leq d_{2}
$$

where $d_{1}>0$ and $d_{2}>0$ denote the lower and upper bounds of the delays, respectively. We define the delay interval as $d_{12}=d_{2}-d_{1}$.

In the system $(1), w(k)$ is a random process with standard one dimension on a probability space $(\Omega, \mathscr{F}, \mathscr{P})$, where $\Omega$ denotes the sample space, and $\mathscr{P}$ is the probability measure on $\mathscr{F}$, which is the $\sigma$-algebra of subsets of the sample space. The sequence of $w(k)$ is generated by $(w(k))_{k \in \mathbf{N}}$, where $\mathbf{N}$ is the set of natural numbers, such that $\mathbf{E}\{w(k)\}=0, \mathbf{E}\left\{w(k)^{2}\right\}=$ 1 , and $\mathbf{E}\{w(i) w(j)\}=0$ for $i \neq j$.

The exogenous disturbance $v(k) \in \mathbb{R}^{q}$ is assumed to belong to $\mathscr{L}_{E_{2}}\left([0, \infty) ; \mathbb{R}^{q}\right)$, which denotes the space of $k$-dimensional nonanticipatory square-integrable process $f(\cdot)=(f(k))_{k \in \mathbf{N}}$ on $\mathbf{N}$ with respect to $\left(\mathscr{F}_{k \in \mathbf{N}}\right)$, such that

$$
\|f\|_{E_{2}}^{2}=\mathbf{E}\left\{\sum_{k=0}^{\infty}\|f(k)\|^{2}\right\}=\sum_{k=0}^{\infty} \mathbf{E}\left\{\|f(k)\|^{2}\right\}<\infty .
$$

The defuzzified output of the T-S fuzzy system (1) is inferred as

$$
\begin{gathered}
\sum: x(k+1)=\sum_{i=1}^{r} h_{i}\left(\theta_{k}\right)\left\{A_{i} x(k)+A_{d i} x\left(k-\tau_{k}\right)+B_{i} v(k)\right. \\
\left.+\left[E_{i} x(k)+G_{i} v(k)\right] w(k)\right\}, \\
y(k)=\sum_{i=1}^{r} h_{i}\left(\theta_{k}\right)\left\{\phi\left(C_{i} x(k)\right)+D_{i} v(k)\right\}, \\
z(k)=\sum_{i=1}^{r} h_{i}\left(\theta_{k}\right) L_{i} x(k),
\end{gathered}
$$


where

$$
h_{i}\left(\theta_{k}\right)=\frac{\mu_{i}\left(\theta_{k}\right)}{\sum_{j=1}^{r} \mu_{j}\left(\theta_{k}\right)}, \quad \mu_{i}\left(\theta_{k}\right)=\prod_{i=1}^{s} F_{j}^{i}\left(\theta_{j k}\right) .
$$

Then, we have $\mu_{i}\left(\theta_{k}\right) \geq 0,(i=1,2, \ldots, r)$ and $\sum_{i=1}^{r} \mu_{i}\left(\theta_{k}\right)>$ 0 , which implies that $h_{i}\left(\theta_{k}\right) \geq 0(i=1,2, \ldots, r)$ and $\sum_{i=1}^{r} h_{i}\left(\theta_{k}\right)=1$.

In this paper, the sensor saturation is described by the saturation function $\phi(\cdot): \mathbb{R}^{m} \rightarrow \mathbb{R}^{m}$ satisfying $\phi \in\left[K_{1}, K_{2}\right]$, for some given diagonal matrices $K_{1} \geq 0, K_{2} \geq 0$ with $K_{2}>K_{1}$, such that

$$
\left(\phi(\zeta)-K_{1} \zeta\right)^{T}\left(\phi(\zeta)-K_{2} \zeta\right) \leq 0, \quad \forall \zeta \in \mathbb{R}^{m}
$$

Furthermore, the nonlinear function $\phi(\zeta)$ can be decomposed into a linear and a nonlinear part

$$
\phi(\zeta)=\phi_{s}(\zeta)+K_{1} \zeta
$$

where the nonlinearity $\phi_{s}(\zeta)$ belongs to $\Phi_{s}$, where

$$
\Phi_{s}=\left\{\phi_{s}: \phi_{s}^{T}(\zeta)\left(\phi_{s}(\zeta)-K \zeta\right) \leq 0\right\}, \quad K \triangleq K_{2}-K_{1} .
$$

For the fuzzy system (1), we construct the following filter with full order to estimate the system states $x(k)$ from measured output $y(k)$ :

$$
\mathscr{F}:\left\{\begin{array}{l}
\widehat{x}(k+1)=\sum_{i=1}^{r} h_{i}\left(\theta_{k}\right)\left\{A_{\mathrm{Fi}} \widehat{x}(k)+B_{\mathrm{Fi}} y(k)\right\}, \\
\widehat{z}(k)=\sum_{i=1}^{r} h_{i}\left(\theta_{k}\right) L_{i} \widehat{x}(k),
\end{array}\right.
$$

where $\widehat{x}(k) \in \mathbb{R}^{n}$ is the filter state vector, $\widehat{z}(k) \in \mathbb{R}^{p}$ is the estimate of $z(k)$, and $A_{F} \in \mathbb{R}^{n \times n}$ and $B_{F} \in \mathbb{R}^{n \times m}$ are the filter gains to be determined.

Define the following error variables:

$$
\begin{gathered}
e_{x}(k)=x(k)-\widehat{x}(k), \quad e(k)=\left[\begin{array}{ll}
x^{T}(k) & e_{x}^{T}(k)
\end{array}\right]^{T}, \\
e_{z}(k)=z(k)-\widehat{z}(k) .
\end{gathered}
$$

Combining the system (1) and the filter (9) yields the filtering error system as follows:

$$
\mathscr{E}:\left\{\begin{aligned}
& e(k+1)=\sum_{i=1}^{r} \sum_{j=1}^{r}\{\left\{\bar{A}_{i j} e(k)+\bar{A}_{d i} I_{K} e\left(k-\tau_{k}\right)\right. \\
&+\bar{B}_{i j} v(k)\left[\bar{E}_{i} I_{K} e(k)+\bar{G}_{i} v(k)\right] w(k) \\
&\left.+\bar{B}_{\mathrm{Fi}} \phi\left(C_{j} e(k)\right)\right\}, \\
& e_{z}(k)=\sum_{i=1}^{r} h_{i}\left(\theta_{k}\right) \bar{L}_{i} e(k),
\end{aligned}\right.
$$

where

$$
\begin{gathered}
\bar{A}_{i j}=\left[\begin{array}{cc}
A_{i} & 0 \\
A_{i}-A_{\mathrm{Fi}}-B_{\mathrm{Fi}} K_{1} C_{j} & A_{\mathrm{Fi}}
\end{array}\right], \quad \bar{A}_{d i}=\left[\begin{array}{c}
A_{d i} \\
A_{d i}
\end{array}\right], \\
\bar{B}_{i j}=\left[\begin{array}{c}
B_{i} \\
B_{i}-B_{\mathrm{Fi}} D_{j}
\end{array}\right], \quad \bar{E}_{i}=\left[\begin{array}{c}
E_{i} \\
E_{i}
\end{array}\right], \quad \bar{G}_{i}=\left[\begin{array}{l}
G_{i} \\
G_{i}
\end{array}\right], \\
\bar{B}_{\mathrm{Fi}}=\left[\begin{array}{c}
0 \\
-B_{\mathrm{Fi}}
\end{array}\right], \quad \bar{C}_{j}=\left[\begin{array}{ll}
C_{j} & 0
\end{array}\right], \\
\bar{L}_{i}=\left[\begin{array}{ll}
0 & L_{i}
\end{array}\right], \quad I_{K}=\left[\begin{array}{ll}
I_{n} & 0
\end{array}\right] .
\end{gathered}
$$

Furthermore, the following definitions will be used in this paper.

Definition 1 (see [32]). For the system in (1) with $\omega(k)=0$, the system is stochastically stable if for any $\psi(k) \in \mathbb{R}^{n}$,

$$
\mathbf{E}\left\{\sum_{k=0}^{\infty}\|x(k)\|^{2}\right\} \leq \theta \mathbf{E}\left\{\|x(0)\|^{2}\right\} .
$$

Definition 2 (see [33]). A mapping $\mathbf{G}: u(k) \rightarrow y(k)$ is said to be input-output stable in the mean square if there exists $\gamma \geq 0$, such that

$$
\|y(k)\|_{E_{2}}=\|\mathbf{G}(u)\|_{E_{2}} \leq \gamma\|u(k)\|_{E_{2}} .
$$

Then, the main objective of this paper is as follows.

$\mathscr{H}_{\infty}$ Filtering Problem. Given a scalar $\gamma>0$, the gains $A_{\mathrm{Fi}}$ and $B_{\mathrm{Fi}}$ of the filter (9) are designed such that for any time-delay $\tau(k)$ satisfying (2), the filter error system (11) is stochastically stable under $v(k)=0$, and for any given integer $\gamma$, the following $\mathscr{H}_{\infty}$ performance index

$$
\|z(k)-\widehat{z}(k)\|_{E_{2}} \leq \gamma\|v(k)\|_{E_{2}}
$$

holds for any function $\phi(\cdot) \in\left[K_{1}, K_{2}\right]$.

\section{Filtering Performance Analysis}

In this section, we focus on the filter performance analysis based on the developed SSG theorem. By the developed SSG theorem to the stochastic systems and a new model transformation to the system $\mathscr{E}$ in (11), the sufficient conditions are proposed, under which the system $\mathscr{E}$ is stochastically stable with a prescribed $\mathscr{H}_{\infty}$ performance index.

\subsection{SSG Theorem for Stochastic Systems}

Lemma 3 (stochastic small gain theorem [34]). Consider the following interconnected system consisting of two subsystems in Figure 1:

$$
\mathcal{S}_{1}: \varepsilon(t)=\mathbf{G} \epsilon(t), \quad \mathcal{S}_{2}: \epsilon(t)=\Delta \varepsilon(t) .
$$

Then, the closed loop systems are input-output stable in mean square for all the subsystems $\mathbf{G}$ and $\Delta$ satisfying

$$
\begin{array}{ll}
\left\|\mathbf{G}\left(u_{1}\right)\right\|_{E_{2}} \leq k_{1}\left\|u_{1}\right\|_{E_{2}}, & k_{1}>0, \\
\left\|\Delta\left(u_{2}\right)\right\|_{E_{2}} \leq k_{2}\left\|u_{2}\right\|_{E_{2}}, & k_{2}>0
\end{array}
$$

if $k_{1} k_{2}<1$ 


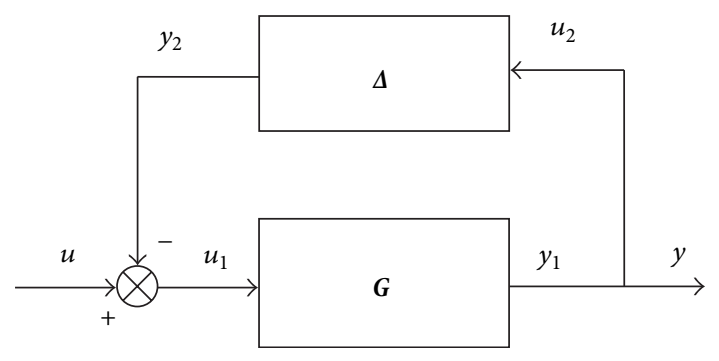

FIGURE 1: The interconnection subsystems.

Proof. Considering (16) yields

$$
\begin{aligned}
\|y\|_{E_{2}} & \leq k_{1}\left\|u_{1}\right\|_{E_{2}}=k_{1}\|u-\Delta(y)\|_{E_{2}} \\
& \leq k_{1}\left(\|u\|_{E_{2}}+k_{2}\|y\|_{E_{2}}\right) .
\end{aligned}
$$

Obviously,

$$
\|y\|_{E_{2}} \leq \frac{k_{1}}{1-k_{1} k_{2}}\|u\|_{E_{2}}
$$

which implies that the closed loop is input-output stable in mean square.

Corollary 4 (stochastic SSG theorem [34]). Consider the system described in (16). The system is input-output stable in mean square if $\left\|T_{\epsilon} \circ \mathbf{G} \circ T_{\varepsilon}\right\|<1$ holds for some nonsingular matrices $T_{y}$ and $T_{\delta}$ with $\left\|T_{\varepsilon} \circ \Delta \circ T_{\epsilon}\right\| \leq 1$.

The proof of Corollary 4 can be readily obtained by following the similar lines of Lemma 3.

3.2. A New Model Transformation. To transform the systems (11) into the form of (16), we express $x\left(k-\tau_{k}\right)$ as

$$
x\left(k-\tau_{k}\right)=\frac{1}{2} x\left(k-d_{1}\right)+\frac{1}{2} x\left(k-d_{2}\right)+\frac{d_{12}}{2} \epsilon(k),
$$

where $(1 / 2)\left[x\left(k-d_{1}\right)+x\left(k-d_{2}\right)\right]$ is the approximation of $x\left(k-\tau_{k}\right)$, and $\left(d_{12} / 2\right) \epsilon(k)$ denotes the approximation error. Then, the system $\mathscr{E}$ can be rewritten as follows:

$$
\mathscr{E}:\left\{\begin{aligned}
e(k+1)=\sum_{i=1}^{r} \sum_{j=1}^{r}\{ & \bar{A}_{i j} e(k)+\frac{1}{2} \bar{A}_{d i} \\
& \times\left[x\left(k-d_{1}\right)+x\left(k-d_{2}\right)+d_{12} \epsilon(k)\right] \\
& +\bar{B}_{i j} v(k)+\left[\bar{E}_{i} I_{K} e(k)+\bar{G}_{i} v(k)\right] \\
& \left.\times w(k),+\bar{B}_{\mathrm{Fi}} \phi\left(C_{j} e(k)\right)\right\}, \\
e_{z}(k)=\sum_{i=1}^{r} h_{i}\left(\theta_{k}\right) & \bar{L}_{i} e(k) .
\end{aligned}\right.
$$

Define $\varepsilon(k)=\mathbf{E}\{x(k+1)\}-x(k)$, and we have

$$
\begin{aligned}
\epsilon(k) & =\frac{2}{d_{12}}\left[x\left(k-\tau_{k}\right)-\frac{1}{2} x\left(k-d_{1}\right)-\frac{1}{2} x\left(k-d_{2}\right)\right] \\
& =\frac{1}{d_{12}}\left[\sum_{i=k-d_{2}}^{k-\tau_{k}-1} \varepsilon(i)-\sum_{i=k-\tau_{k}}^{k-d_{1}-1} \varepsilon(i)\right] \\
& =\frac{1}{d_{12}}\left[\sum_{i=k-d_{2}}^{k-d_{1}-1} \beta(i) \varepsilon(i)\right],
\end{aligned}
$$

where

$$
\beta(i) \triangleq \begin{cases}1, & \text { when } i \leqslant k-\tau_{k}-1 \\ -1, & \text { when } i>k-\tau_{k}-1\end{cases}
$$

Then, obviously the system (21) can be rewritten in the form as follows:

$$
\mathscr{E}_{1}: \sigma(k)=\sum_{i=1}^{r} \sum_{j=1}^{r} \mathbf{G} \xi(k), \quad \mathscr{E}_{2}: \epsilon(k)=\Delta \varepsilon(k)
$$

where

$$
\begin{aligned}
& \xi(k)=\left[\begin{array}{llllll}
e^{T}(k) & x^{T}\left(k-d_{1}\right) & x^{T}\left(k-d_{2}\right) & \epsilon(k) & v(k) & \phi_{s}\left(C_{j} x(k)\right)
\end{array}\right]^{T}, \\
& \sigma(k)=\left[\begin{array}{lll}
e^{T}(k+1) & \varepsilon^{T}(k) & e_{z}^{T}(k)
\end{array}\right]^{T}, \quad \mathbf{G}=\left[\begin{array}{lll}
\mathbf{G}_{1}^{T} & \mathbf{G}^{T} & \mathbf{G}_{3}^{T}
\end{array}\right]^{T},
\end{aligned}
$$

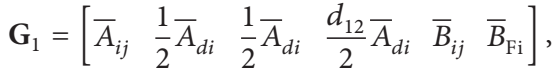

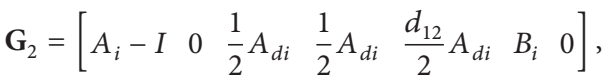

$$
\begin{aligned}
& \mathbf{G}_{3}=\left[\begin{array}{ll}
\bar{L}_{i} & 0_{p \times(3 n+m+q)}
\end{array}\right] \text {. }
\end{aligned}
$$

Furthermore, the SSG of the mapping $\Delta$ denoted by $\gamma\left(\Delta_{T}\right)$ has an upper bound.

Lemma 5. $\gamma\left(\Delta_{T}\right)$ has an upper bound

$$
\gamma\left(\Delta_{T}\right)=\sup \frac{\|T \epsilon(k)\|_{E_{2}}}{\|T \varepsilon(k)\|_{E_{2}}} \leq 1
$$

Proof. According to the fact that $|\beta(i)|=1$, by using Jensen's inequality, it can be derived under zero initial condition

$$
\begin{aligned}
\|T \epsilon(k)\|_{E_{2}}^{2} & =\mathbf{E}\left\{\sum_{j=0}^{\infty}\left[\epsilon^{T}(j) Z \epsilon(j)\right]\right\} \\
& \leq \frac{2}{d_{12}^{2}} \mathbf{E}\left\{\sum_{i=0}^{\infty}\left[d_{12} \sum_{j=i-d_{2}}^{i-d_{1}-1} \beta^{2}(j) \epsilon^{T}(j) \epsilon(j)\right]\right\}
\end{aligned}
$$




$$
\begin{aligned}
& =\frac{1}{d_{12}} \mathbf{E}\left\{\sum_{j=-d_{2}}^{-d_{1}-1} \sum_{i=0}^{\infty} \epsilon^{T}(i+j) \epsilon(i+j)\right\} \\
& \leq \frac{1}{d_{12}} \mathbf{E}\left\{\sum_{j=-d_{2}} \sum_{i=0}^{-d_{1}-1} \epsilon^{T}(i) \epsilon(i)\right\} \\
& \leq \mathbf{E}\left\{\sum_{j=0}^{\infty}\left[\varepsilon^{T}(j) Z \varepsilon(j)\right]\right\}=\|T \varepsilon(k)\|_{E_{2}}^{2},
\end{aligned}
$$

where $Z=T^{T} T$, which implies $\gamma\left(\Delta_{T}\right) \leq 1$. The proof is completed.

From Lemmas 3 and 5, the system $\mathscr{E}$ in (11) is input-output stable in mean square if the SSG of the subsystem $\mathscr{E}_{1}$ satisfies the conditions in Lemma 3, which will be investigated in the following section.

3.3. $\mathscr{H}_{\infty}$ Performance Analysis. In this section, the $\mathscr{H}_{\infty}$ performance of the filtering error system is analyzed by the SSG of the subsystem $\mathscr{E}_{1}$ in (24) in order to establish the conditions under which the $\mathscr{H}_{\infty}$ performance index is guaranteed for the filtering error system (11).

Theorem 6. Suppose that the system matrices $A_{i}, A_{d i}, B_{i}, C_{i}$, $D_{i}$, and $L_{i}$ represent a fixed system. Given the filter matrices $A_{F i}, B_{F i}$ and integers $d_{2}>d_{1} \geq 0$ and a constant $\gamma>0$, the filtering error system (21) is input-output stable in mean square with an $\mathscr{H}_{\infty}$ performance attention level $\gamma$ for any timevarying delay satisfying (2) if there exist matrices $P>0, Q_{i}>$ $0, i=1,2$, such that

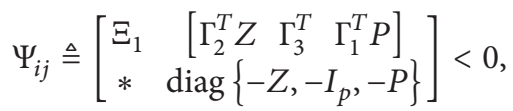

where

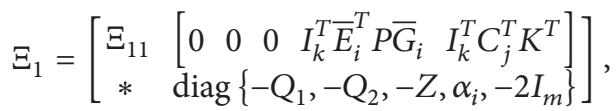

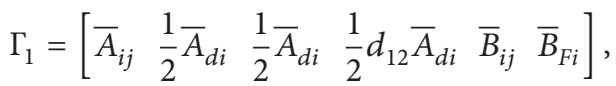

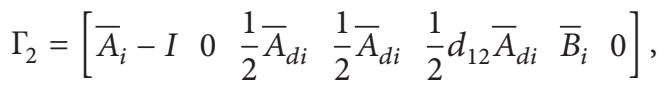

$$
\begin{aligned}
& \Gamma_{3}=\left[\begin{array}{ll}
\bar{L}_{i} & 0_{p \times(3 n+m+q)}
\end{array}\right], \quad \alpha_{i}=-\gamma^{2} I_{q}+\bar{G}_{i}^{T} P \bar{G}_{i}, \\
& \Xi_{11}=-P+I_{K}^{T}\left(Q_{1}+Q_{2}+\bar{E}_{i}^{T} P \bar{E}_{i}\right) I_{K} \text {. }
\end{aligned}
$$

Proof. Choose the following Lyapunov-Krasovskii functional:

$$
V(k)=V_{1}(k)+V_{2}(k)
$$

with

$$
\begin{gathered}
V_{1}(k)=e^{T}(k) P e(k), \\
V_{2}(k)=\sum_{i=k-d_{1}}^{k-1} x^{T}(i) Q_{1} x(i)+\sum_{i=k-d_{2}}^{k-1} x^{T}(i) Q_{2} x(i),
\end{gathered}
$$

where $P>0$ and $Q_{i}>0, i=1,2$ are the matrices to be determined.

Define $\Delta V(k)=\mathbf{E}\{V(k+1)\}-V(k)$, then we have

$$
\begin{aligned}
& \Delta V_{1}(k) \\
& =\mathbf{E}\left\{e^{T}(k+1) P e(k+1)\right\}-e^{T}(k) P e(k) \\
& \leq \sum_{i=1}^{r} \sum_{j=1}^{r}\left\{\left(\begin{array}{c}
\xi^{T}(k) \Gamma_{1}^{T} P \Gamma_{1} \xi(k)-e^{T}(k) P e(k) \\
-2 \phi_{s}\left(C_{j} x(k)\right)\left[\phi_{s}\left(C_{j} x(k)\right)-K C_{j} x(k)\right]
\end{array}\right)\right\}, \\
& \begin{aligned}
\Delta V_{2}(k)= & x^{T}(k) Q_{1} x(k)-x^{T}\left(k-d_{1}\right) Q_{1} x\left(k-d_{1}\right) \\
& +x^{T}(k) Q_{2} x(k)-x^{T}\left(k-d_{2}\right) Q_{2} x\left(k-d_{2}\right) .
\end{aligned}
\end{aligned}
$$

Then, define

$$
\begin{array}{r}
J_{s}=\mathbf{E}\left\{\sum _ { k = 0 } ^ { \infty } \left[\varepsilon^{T}(k) Z \varepsilon(k)-\epsilon^{T}(k) Z \epsilon(k)\right.\right. \\
\left.\left.+e_{z}^{T}(k) e_{z}(k)-\gamma^{2} v^{T}(k) v(k)\right]\right\},
\end{array}
$$

which together with (30) yields

$$
\begin{aligned}
& J_{s} \leq J_{s}+V(\infty)-V(0) \\
& =\mathbf{E}\left\{\sum_{k=0}^{\infty}\left(\begin{array}{c}
\Delta V(k)+\varepsilon^{T}(k) Z \varepsilon(k)-\epsilon^{T}(k) Z \epsilon(k) \\
+e_{z}^{T}(k) e_{z}(k)-\gamma^{2} v^{T}(k) v(k)
\end{array}\right)\right\} \\
& =\mathbf{E}\left\{\sum_{k=0}^{\infty} \sum_{i=1}^{r} \sum_{j=1}^{r}\left[\xi^{T}(k)\left(\begin{array}{c}
\Xi_{1}+\Gamma_{1}^{T} P \Gamma_{1} \\
+\Gamma_{2}^{T} Z \Gamma_{2}+\Gamma_{3}^{T} \Gamma_{3}
\end{array}\right) \xi(k)\right]\right\} .
\end{aligned}
$$

By the Schur complement, it can be verified that $J_{s}<0$ is guaranteed by $\Psi_{i j}<0$, which implies that if $Z=T^{T} T$, we have $\gamma\left(\mathbf{G}_{T}\right)<1$, where $\mathbf{G}$ is defined in (24). According to Lemmas 3 and 5, it is shown that the system $\mathscr{E}$ in (11) is input-output stable in mean square.

Furthermore, $J_{s}<0$ implies that

$$
\|T \varepsilon(k)\|_{E_{2}}^{2}+\left\|e_{z}(k)\right\|_{E_{2}}^{2}<\|T \epsilon(k)\|_{E_{2}}^{2}+\gamma^{2}\|v(k)\|_{E_{2}}^{2},
$$

which together with (30) yields $\left\|e_{z}(k)\right\|_{E_{2}}^{2}<\gamma^{2}\|v(k)\|_{E_{2}}^{2}$. The proof is completed.

Theorem 6 shows the conditions under which the system $\mathscr{E}$ in (11) is input-output stable in mean square with a prescribed $\mathscr{H}_{\infty}$ performance index. Moreover, the conditions for stochastic stability of the system $\mathscr{E}$ can be reached by the Lyapunov-Krasovskii functional method, which will be discussed in the following theorem. 
Theorem 7. For the forward system $\mathscr{E}$ in (24), if Theorem 6 holds, then, the system $\mathscr{E}$ is stochastically stable for $v(k)=0$.

Proof. For the LKF defined in (30), we have

$$
\begin{aligned}
\Delta V_{c}(k) & =\Delta V(k)+\varepsilon^{T}(k) Z \varepsilon(k)-\epsilon^{T}(k) Z \epsilon(k) \\
& =\xi_{1}^{T}(k)\left(\widetilde{\Xi}_{1}+\widetilde{\Gamma}_{1}^{T} P \widetilde{\Gamma}_{1}+\widetilde{\Gamma}_{2}^{T} Z \widetilde{\Gamma}_{2}\right) \xi_{1}(k),
\end{aligned}
$$

with

$$
\begin{aligned}
& \xi_{1}(k)=\left[\begin{array}{lllll}
e^{T}(k) & x^{T}\left(k-d_{1}\right) & x^{T}\left(k-d_{2}\right) & \epsilon(k) & \phi_{s}\left(C_{j} x(k)\right)
\end{array}\right]^{T}, \\
& \widetilde{\Xi}_{1}=\left[\begin{array}{cc}
\Xi_{11} & {\left[\begin{array}{cccc}
0 & 0 & 0 & I_{k}^{T} C_{j}^{T} K^{T}
\end{array}\right]} \\
* & \operatorname{diag}\left\{-Q_{1},-Q_{2},-Z,-2 I_{m}\right\}
\end{array}\right],
\end{aligned}
$$

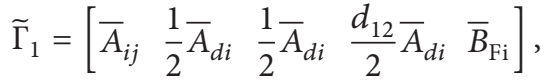

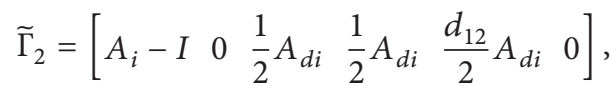

where $\Xi_{11}$ is defined in (28).

Define $\left(\widetilde{\Xi}_{1}+\widetilde{\Gamma}_{1}^{T} P \widetilde{\Gamma}_{1}+\widetilde{\Gamma}_{2}^{T} Z \widetilde{\Gamma}_{2}\right)$ as $\Phi_{i j}$. It can be shown that $\Phi_{i j}<0$, if Theorem 6 holds. Then, we can always find a small scalar $\lambda>0$, such that

$$
\Phi_{i j}<\left[\begin{array}{cc}
-\lambda I_{n} & 0 \\
0 & 0
\end{array}\right]
$$

which implies that

$$
\mathbf{E}\left\{V_{c}(k+1)-V_{c}(k)\right\} \leq-\lambda x^{T}(k) x(k)<0 .
$$

For any positive integer $R$, by summing up the inequality on both sides from $k=0, \ldots, R$, we obtain

$$
\mathbf{E}\left\{V_{c}(R)-V_{c}(0)\right\} \leq-\lambda \sum_{k=0}^{R} x^{T}(k) x(k)<0,
$$

which implies that when $R \rightarrow \infty$,

$$
\begin{aligned}
\sum_{k=0}^{\infty} \mathbf{E}\left\{x^{T}(k) x(k)\right\} & \leq \frac{1}{\lambda} \mathbf{E}\left\{V_{c}(0)-V_{c}(\infty)\right\} \\
& \leq \frac{1}{\lambda} \mathbf{E}\left\{V_{c}(0)\right\}<\theta \mathbf{E}\left\{\|x(0)\|^{2}\right\},
\end{aligned}
$$

which indicates that the systems in (24) are stochastically stable according to Definition 1 . The proof is completed.

Remark 8. It is well known that free-weighting matrices are introduced a lot to estimate the upper bound of the term $\left(1 / d_{12}\right) \sum_{i=-d_{2}}^{-d_{1}-1} \epsilon^{T}(k+i) Z \epsilon(k+i)$, while in this paper, Jensen's inequality is employed instead of such weighting matrices, which reduce the NoV of the proposed conditions to $3 n^{2}+3 n$. It is remarkably smaller than the results in some existing literatures, such as $9 n^{2}+3 n$ [35], and $11 n^{2}+2.5 n$ [16], $13 n^{2}+5 n[36]$, and $31 n^{2}+7 n$ [17], which demonstrates the computational advantage of the proposed approach obviously [24].

\section{4. $\mathscr{H}_{\infty}$ Filter Design}

This section is devoted to the $\mathscr{H}_{\infty}$ filter design problem for the system (1) based on Theorem 6. Sufficient conditions for the existence of the $\mathscr{H}_{\infty}$ filter are provided in the following theorem.

Theorem 9. Consider the discrete-time fuzzy stochastic system in (1), for a given constant $\gamma>0$, if there exist matrices $0<X \in$ $\mathbb{R}^{n \times n}, 0<Y \in \mathbb{R}^{n \times n}, \bar{A}_{F i} \in \mathbb{R}^{n \times n}$, and $\bar{B}_{F i} \in \mathbb{R}^{n \times m}$, such that the following LMI holds:

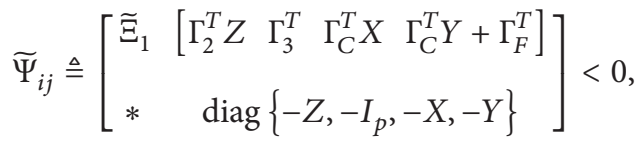

where

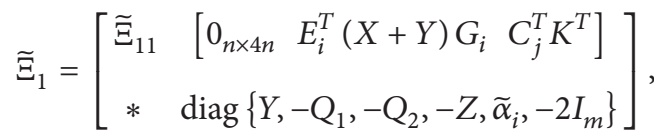

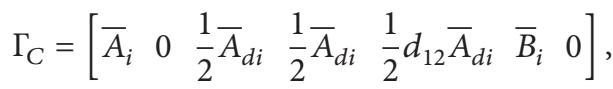

$$
\begin{aligned}
& \Gamma_{F}=\left[\begin{array}{lllll}
-\bar{B}_{F i} K_{1} C_{j}-\bar{A}_{F i} & \bar{A}_{F i} & 0_{n \times 3 n} & -\bar{B}_{F i} D_{j} & -\bar{B}_{F i}
\end{array}\right] \text {, } \\
& \widetilde{\alpha}_{i}=-\gamma^{2} I_{q}+G_{i}^{T}(X+Y) G_{i}, \\
& \widetilde{\Xi}_{11}=-X+Q_{1}+Q_{2}+E_{i}^{T}(X+Y) E_{i},
\end{aligned}
$$

then, the system $\mathscr{E}$ in (11) is stochastically stable with $\mathscr{H}_{\infty}$ performance level $\gamma$, and the filter gains are given by

$$
A_{F i}=Y^{-1} \bar{A}_{F i}, \quad B_{F i}=Y^{-1} \bar{B}_{F i} .
$$

Proof. Define $P$ introduced in Theorem 6 as

$$
P=\left[\begin{array}{cc}
X & 0 \\
0 & Y
\end{array}\right]
$$

where $0<X \in \mathbb{R}^{n \times n}$ and $0<Y \in \mathbb{R}^{n \times n}$ are to be determined such that solvability of (28) is satisfied.

Then, we have

$$
\begin{gathered}
{\left[\begin{array}{cc}
A_{i}^{T} & A_{i}^{T}-A_{\mathrm{Fi}}^{T}-C_{j}^{T} K_{1}^{T} B_{\mathrm{Fi}}^{T} \\
0 & A_{\mathrm{Fi}}^{T}
\end{array}\right]\left[\begin{array}{cc}
X & 0 \\
0 & Y
\end{array}\right]} \\
=\left[\begin{array}{cc}
A_{i}^{T} X & A_{i}^{T} Y-A_{\mathrm{Fi}}^{T} Y-C_{j}^{T} K_{1}^{T} B_{\mathrm{Fi}}^{T} Y \\
0 & A_{\mathrm{Fi}}^{T} Y
\end{array}\right], \\
{\left[\begin{array}{ll}
B_{i}^{T} & B_{i}^{T}-D_{j}^{T} B_{\mathrm{Fi}}^{T}
\end{array}\right]\left[\begin{array}{cc}
X & 0 \\
0 & Y
\end{array}\right]} \\
=\left[\begin{array}{ll}
B_{i}^{T} X & B_{i}^{T} Y-D_{j}^{T} B_{\mathrm{Fi}}^{T} Y
\end{array}\right] .
\end{gathered}
$$

Define $\bar{A}_{\mathrm{Fi}}=Y A_{\mathrm{Fi}}, \bar{B}_{\mathrm{Fi}}=Y B_{\mathrm{Fi}}$, which together with (28) yields (42). The proof is completed. 


\section{Numerical Example}

In this section, an example is provided to demonstrate effectiveness of the filter design method proposed in the preceding sections.

Example 10. Consider the system (1) with the following parameters:

$$
\begin{aligned}
& A_{1}=\left[\begin{array}{ccc}
-0.28 & -0.01 & -0.02 \\
-0.2 & -0.25 & -0.29 \\
0.03 & -0.04 & -0.23
\end{array}\right] \text {, } \\
& A_{d 1}=\left[\begin{array}{ccc}
0.01 & 0.02 & 0 \\
0.01 & 0 & 0.01 \\
0 & 0 & -0.01
\end{array}\right] \text {, } \\
& E_{1}=\left[\begin{array}{ccc}
-0.11 & 0.1 & 1.66 \\
0 & 0.46 & -0.47 \\
0.47 & -0.11 & -0.43
\end{array}\right], \quad B_{1}=\left[\begin{array}{c}
-2.02 \\
3.83 \\
1.39
\end{array}\right] \text {, } \\
& C_{1}=\left[\begin{array}{ccc}
-0.11 & 0.05 & -0.1 \\
0.69 & 0.35 & 0.48
\end{array}\right], \quad L_{1}=\left[\begin{array}{ccc}
0.1 & 0.1 & 0.1 \\
0.12 & 0.1 & 0.19
\end{array}\right] \text {, } \\
& G_{1}=\left[\begin{array}{lll}
-0.21 & 0.61 & 0.15
\end{array}\right]^{T}, \quad D_{1}=\left[\begin{array}{ll}
0.3 & -0.3
\end{array}\right]^{T}, \\
& A_{2}=\left[\begin{array}{ccc}
-0.35 & 0.0 & 0.01 \\
-0.59 & -0.24 & 0.02 \\
0.1 & -0.06 & -0.72
\end{array}\right] \text {, } \\
& A_{d 2}=\left[\begin{array}{ccc}
0.01 & 0.02 & 0 \\
0.01 & 0 & 0.01 \\
0 & 0 & 0.01
\end{array}\right] \text {, } \\
& E_{2}=\left[\begin{array}{ccc}
-0.12 & -0.11 & 0.38 \\
0.11 & 0.64 & -0.18 \\
-0.31 & -0.63 & -0.6
\end{array}\right], \quad B_{2}=\left[\begin{array}{c}
2.04 \\
-3.25 \\
-0.93
\end{array}\right] \text {, } \\
& C_{2}=\left[\begin{array}{ccc}
-0.2 & -0.1 & -0.2 \\
0.5 & 0.2 & 0.21
\end{array}\right], \quad L_{2}=\left[\begin{array}{ccc}
0.2 & 0.19 & 0.2 \\
0.1 & 0.1 & 0.2
\end{array}\right] \text {, } \\
& G_{2}=\left[\begin{array}{lll}
-0.43 & 2.11 & 0.51
\end{array}\right]^{T}, \quad D_{2}=\left[\begin{array}{ll}
0.1 & 0.47
\end{array}\right]^{T},
\end{aligned}
$$

and the saturation parameters are selected as

$$
K_{1}=\left[\begin{array}{cc}
0.6 & 0 \\
0 & 0.7
\end{array}\right], \quad K_{2}=\left[\begin{array}{cc}
0.8 & 0 \\
0 & 0.8
\end{array}\right]
$$

the membership functions are

$$
h_{1}(\theta)=\frac{1-\sin \left(x_{1}\right)}{2}, \quad h_{1}(\theta)=\frac{1+\sin \left(x_{1}\right)}{2},
$$

and the noises $v(k)$ are assumed to be $v(k)=1 /\left(0.1+k^{2}\right)$, which satisfy the constraint in (3), and set $d_{1}=1$ and $d_{2}=2$.

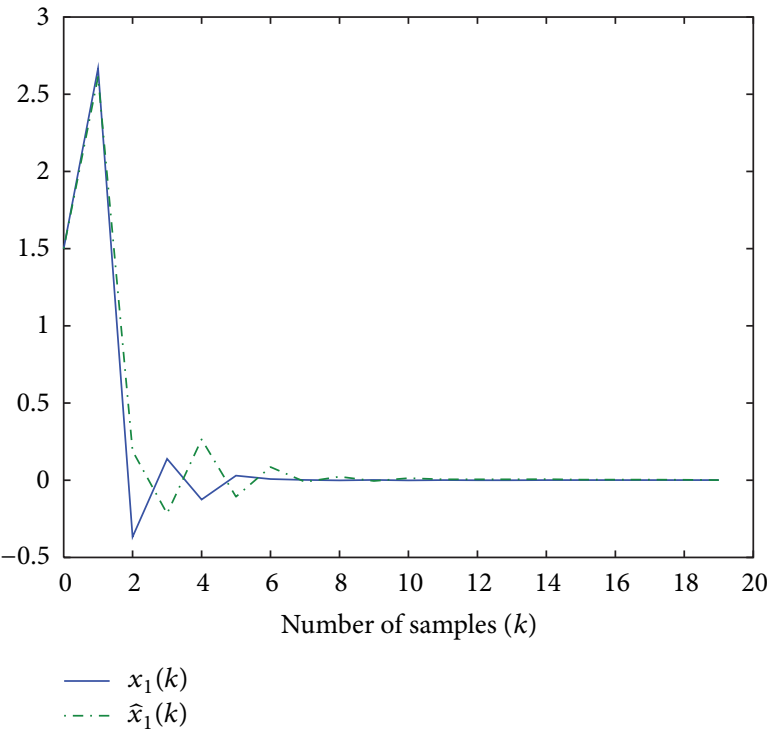

FIGURE 2: $x_{1}(k)$ and $\hat{x}_{1}(k)$.

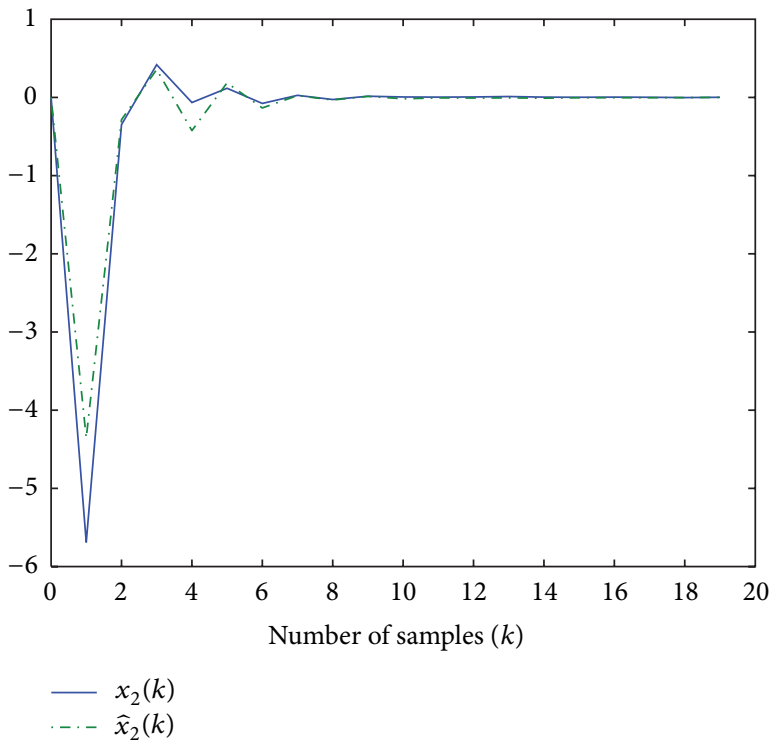

FIGURE 3: $x_{2}(k)$ and $\hat{x}_{2}(k)$.

To design the $\mathscr{H}_{\infty}$ filter, by solving the LMI condition (42) under the prescribed disturbance level $\gamma=1.2$, it can be obtained that

$$
\begin{aligned}
& \bar{A}_{F 1}=\left[\begin{array}{ccc}
-0.0001 & -0.0008 & 0.0021 \\
0.0001 & 0.0005 & -0.0013 \\
0.0000 & 0.0002 & -0.0006
\end{array}\right], \\
& \bar{B}_{F 1}=\left[\begin{array}{cc}
-0.5877 & 1.4425 \\
1.2489 & -2.1186 \\
0.5082 & -0.5446
\end{array}\right],
\end{aligned}
$$




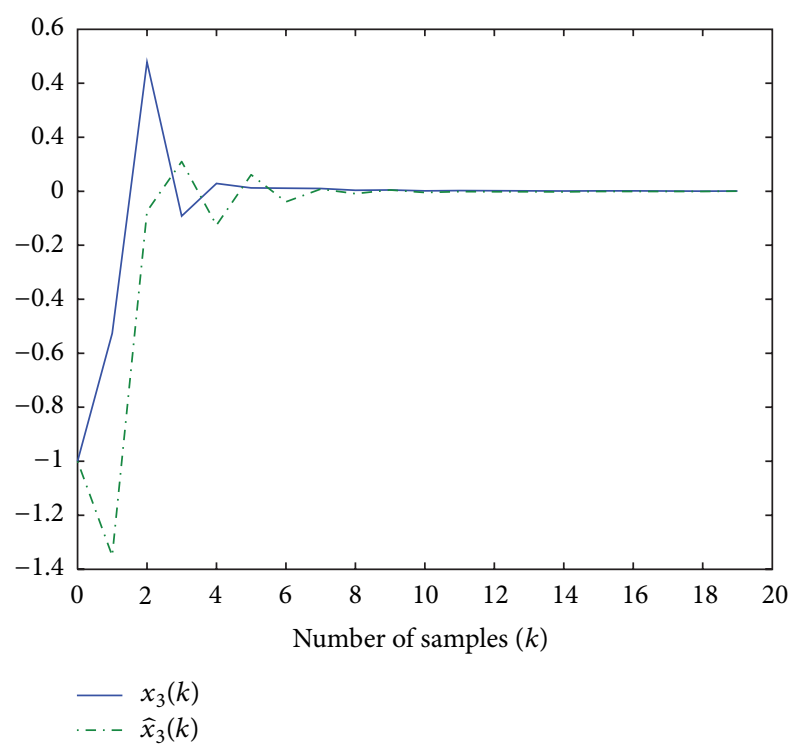

Figure 4: $x_{3}(k)$ and $\hat{x}_{3}(k)$.

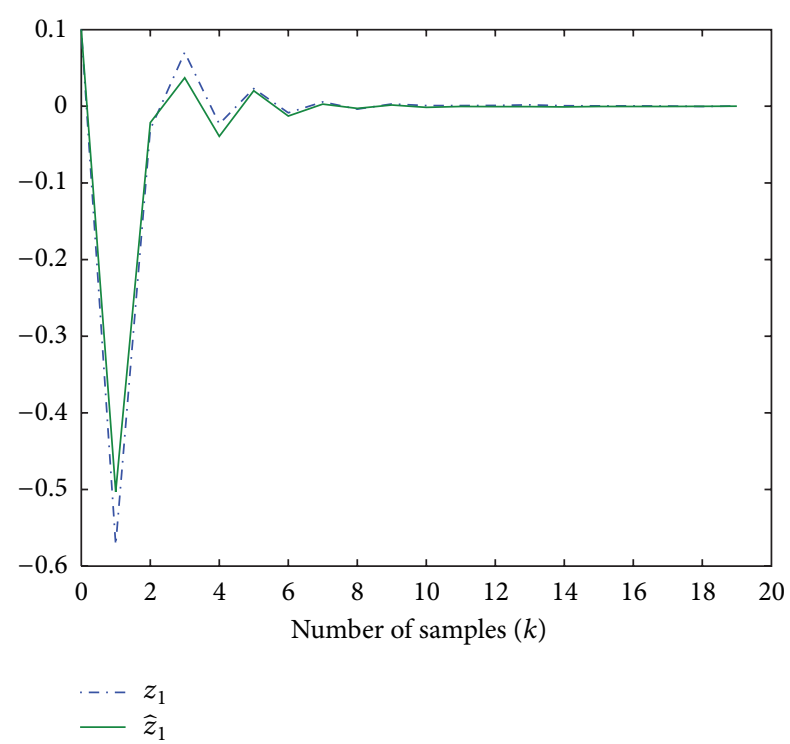

FIGURE 5: $z_{1}(k)$ and $\widehat{z}_{1}(k)$.

$$
\begin{aligned}
& \bar{A}_{F 2}=\left[\begin{array}{ccc}
-0.0002 & -0.0005 & 0.0012 \\
0.0002 & 0.0000 & 0.0002 \\
0.0000 & 0.0001 & -0.0002
\end{array}\right], \\
& \bar{B}_{F 2}=\left[\begin{array}{cc}
1.0949 & 2.0782 \\
-0.9802 & -3.5244 \\
0.0898 & -1.1212
\end{array}\right] .
\end{aligned}
$$

Assume the sensor saturation as

$$
\phi(y)=\frac{K_{1}+K_{2}}{2} y+\frac{K_{2}-K_{1}}{2} \sin (y),
$$

which satisfies (8), and the initial conditions as $x(0)=$ $\left[\begin{array}{lll}1.5 & 0 & -1\end{array}\right]^{T}, x(-1)=x(-2)=\left[\begin{array}{lll}1 & 0.1 & -0.6\end{array}\right]^{T}$. By

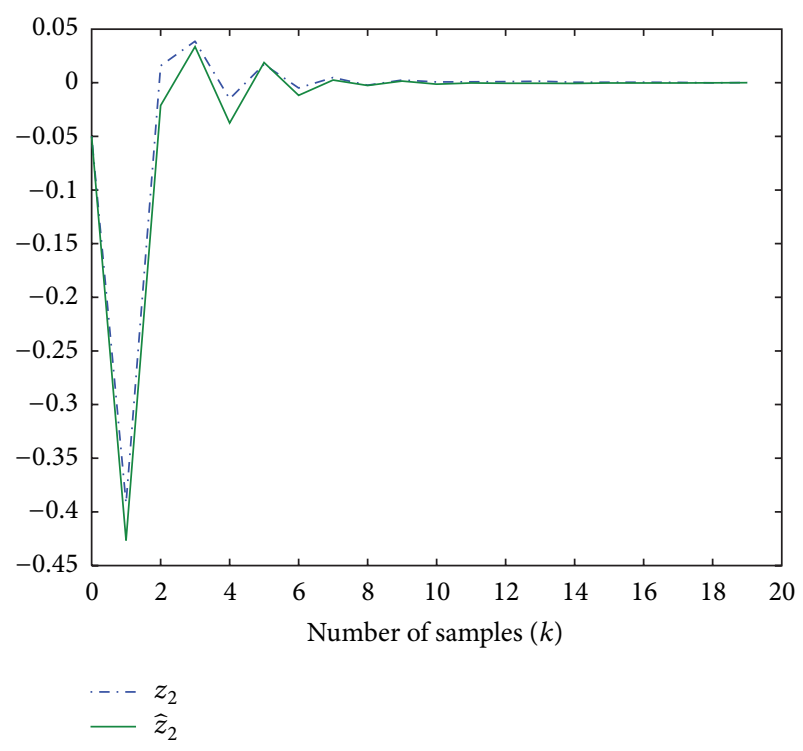

FIGURE 6: $z_{2}(k)$ and $\widehat{z}_{2}(k)$.

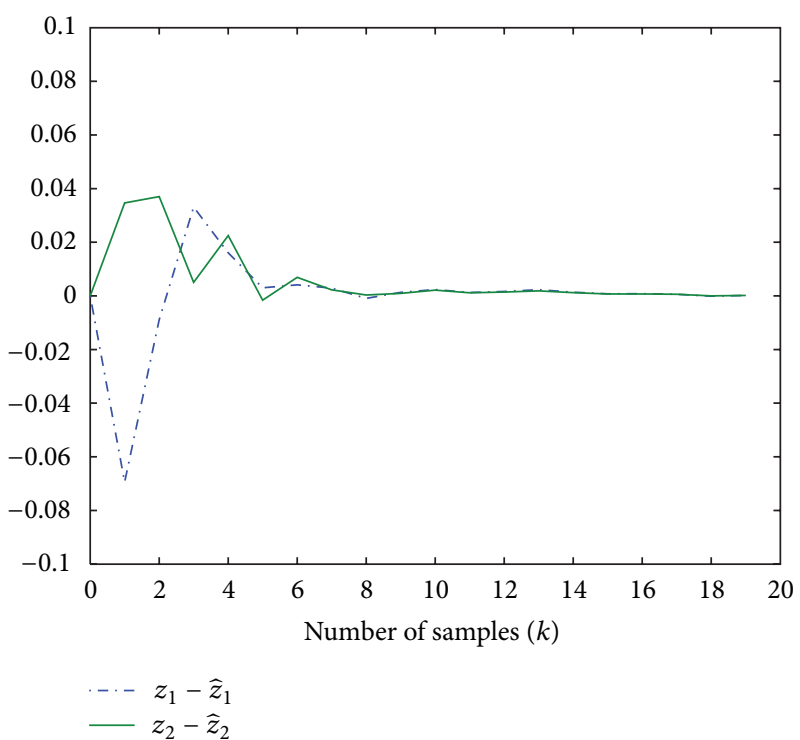

FIGURE 7: $e_{z 1}(k)$ and $e_{z 2}(k)$.

calculation, it can be obtained that the $\left\|e_{z}(k)\right\|_{E_{2}}^{2}=0.0095$ and $\|v(k)\|_{E_{2}}^{2}=3.0810$ that yields $\gamma=0.0555$ which is below the given $\gamma=1.2$, which demonstrates effectiveness of the proposed filter design. In addition, the trajectories of state variable $x(k)$ and its estimate $\widehat{x}(k)$ are shown in Figures 2, 3, and $4, z(k)$ and its estimate $\widehat{z}(k)$ are given in Figures 5 and 6 , and $e_{z}(k)=z(k)-\widehat{z}(k)$ is provided in Figure 7 .

\section{Conclusion}

This paper addresses the $\mathscr{H}_{\infty}$ filtering problem for T-S fuzzy systems subject to sensor saturation. The plant is considered with random noise depending on state and externaldisturbance. The system is transformed into an input-output 
form consisted of two interconnected subsystems. Based on the SSG theorem developed to stochastic systems and the proposed Lyapunov-Krasovskii function, sufficient conditions under which the $\mathscr{H}_{\infty}$ filter can be achieved with the prescribed $\mathscr{H}_{\infty}$ performance index are established. Finally, a numerical example is presented to demonstrate the effectiveness of the proposed filter design scheme.

\section{References}

[1] H. R. Karimi, "Observer-based mixed $\mathrm{H}_{2} / \mathrm{H}_{\infty}$ control design of linear systems with time-varying delays: an LMI approach," International Journal of Control, Automation and Systems, vol. 6, no. 1, pp. 1-14, 2008.

[2] H. R. Karimi and H. Gao, "New delay-dependent exponential $\mathrm{H}_{\infty}$ synchronization for uncertain neural networks with mixed time delays," IEEE Transactions on Systems, Man, and Cybernetics, Part B, vol. 40, no. 1, pp. 173-185, 2010.

[3] M. Liu, D. W. C. Ho, and Y. Niu, "Stabilization of Markovian jump linear system over networks with random communication delay," Automatica, vol. 45, no. 2, pp. 416-421, 2009.

[4] M. Liu, D. W. C. Ho, and Y. Niu, "Robust filtering design for stochastic system with mode-dependent output quantization," IEEE Transactions on Signal Processing, vol. 58, no. 12, pp. 64106416, 2010.

[5] R. Yang, P. Shi, and G.-P. Liu, "Filtering for discrete-time networked nonlinear systems with mixed random delays and packet dropouts," IEEE Transactions on Automatic Control, vol. 56, no. 11, pp. 2655-2660, 2011.

[6] R. Yang, Z. Zhang, and P. Shi, "Exponential stability on stochastic neural networks with discrete interval and distributed delays," IEEE Transactions on Neural Networks, vol. 21, no. 1, pp. 169-175, 2010.

[7] H. R. Karimi and H. Gao, "Mixed $\mathrm{H}_{2} / \mathrm{H}_{\infty}$ output-feedback control of second-order neutral systems with time-varying state and input delays," ISA Transactions, vol. 47, no. 3, pp. 311-324, 2008.

[8] H. R. Karimi, "Optimal vibration control of vehicle engine-body system using Haar functions," International Journal of Control, Automation and Systems, vol. 4, no. 6, pp. 714-724, 2006.

[9] M. J. Wang and M. F. Yang, "Application of signed distances method to integrate linear programming for fuzzy multiobjective project management," International Journal of Innovative Computing, Information and Control, vol. 7, no. 1, pp. 237252, 2011.

[10] H. Li, H. Liu, H. Gao, and P. Shi, "Reliable fuzzy control for active suspension systems with actuator delay and fault," IEEE Transactions on Fuzzy Systems, vol. 20, no. 2, pp. 342-357, 2012.

[11] H. Li, B. Chen, Q. Zhou, and W. Qian, "Robust stability for uncertain delayed fuzzy Hopfield neural networks with Markovian jumping parameters," IEEE Transactions on Systems, Man, and Cybernetics, Part B, vol. 39, no. 1, pp. 94-102, 2009.

[12] T. Niknam, H. D. Mojarrad, and M. Nayeripour, "A new hybrid fuzzy adaptive particle swarm optimization for nonconvex economic dispatch," International Journal of Innovative Computing, Information and Control, vol. 7, no. 1, pp. 189-202, 2011.

[13] F. Li and X. Zhang, "Delay-range-dependent robust $H_{\infty}$ filtering for singular LPV systems with variant delay," International Journal of Innovative Computing, Information and Control, vol. 9, no. 1, pp. 339-353, 2013.
[14] T.-C. Lin, S.-W. Chang, and C.-H. Hsu, "Robust adaptive fuzzy sliding mode control for a class of uncertain discretetime nonlinear systems," International Journal of Innovative Computing, Information and Control, vol. 8, no. $1 \mathrm{~A}$, pp. 347359, 2012.

[15] H. Gao and C. Wang, "A delay-dependent approach to robust $H_{\infty}$ filtering for uncertain discrete-time state-delayed systems," IEEE Transactions on Signal Processing, vol. 52, no. 6, pp. 16311640, 2004.

[16] H. Gao and T. Chen, "New results on stability of discrete-time systems with time-varying state delay," IEEE Transactions on Automatic Control, vol. 52, no. 2, pp. 328-334, 2007.

[17] X. Meng, J. Lam, B. Du, and H. Gao, "A delay-partitioning approach to the stability analysis of discrete-time systems," Automatica, vol. 46, no. 3, pp. 610-614, 2010.

[18] X. Su, P. Shi, L. Wu, and Y. D. Song, "A novel control design on discrete-time takagisugeno fuzzy systems with time-varying delays," IEEE Transactions on Fuzzy Systems, no. 99, p. 9, 2012.

[19] Y.-P. Huang and K. Zhou, "Robust stability of uncertain timedelay systems," IEEE Transactions on Automatic Control, vol. 45, no. 11, pp. 2169-2173, 2000.

[20] C.-Y. Kao and B. Lincoln, "Simple stability criteria for systems with time-varying delays," Automatica, vol. 40, no. 8, pp. 14291434, 2004.

[21] H. R. Karimi, M. Zapateiro, and N. Luo, "Stability analysis and control synthesis of neutral systems with time-varying delays and nonlinear uncertainties," Chaos, Solitons \& Fractals, vol. 42, no. 1, pp. 595-603, 2009.

[22] X. M. Zhang and Q. L. Han, "Delay-dependent robust $H_{\infty}$ filtering for uncertain discrete-time systems with time-varying delay based on a finite sum inequality," IEEE Transactions on Circuits and Systems II, vol. 53, no. 12, pp. 1466-1470, 2006.

[23] K. Gu, Y. Zhang, and S. Xu, "Small gain problem in coupled differential-difference equations, time-varying delays, and direct Lyapunov method," International Journal of Robust and Nonlinear Control, vol. 21, no. 4, pp. 429-451, 2011.

[24] X. Li and H. Gao, "A new model transformation of discrete-time systems with time-varying delay and its application to stability analysis," IEEE Transactions on Automatic Control, vol. 56, no. 9, pp. 2172-2178, 2011.

[25] L. Wu, X. Su, P. Shi, and J. Qiu, "Model approximation for discrete-time state-delay systems in the T-S fuzzy framework," IEEE Transactions on Fuzzy Systems, vol. 19, no. 2, pp. 366-378, 2011.

[26] L. Wu, X. Su, P. Shi, and J. Qiu, "A new approach to stability analysis and stabilization of discrete-time T-S fuzzy timevarying delay systems," IEEE Transactions on Systems, Man, and Cybernetics, Part B, vol. 41, no. 1, pp. 273-286, 2011.

[27] W. H. Ho, S. H. Chen, I. T. Chen, J. H. Chou, and C. C. $\mathrm{Shu}$, "Design of stable and quadraticoptimal static output feedback controllers for TS-fuzzy-model-based control systems: an integrative computational approach," International Journal of Innovative Computing, Information and Control, vol. 20, no. 6, pp. 403-418, 2012.

[28] L. Wu and Z. Wang, "Fuzzy filtering of nonlinear fuzzy stochastic systems with time-varying delay," Signal Processing, vol. 89, no. 9, pp. 1739-1753, 2009.

[29] D. Zhang, H. Wang, B. Lu, and Z. Wang, "LMI-based fault detection fuzzy observer design with multiple performance constraints for a class of non-linear systems: comparative study," International Journal of Innovative Computing, Information and Control, vol. 8, no. 1, pp. 633-645, 2012. 
[30] X. Su, L. Wu, and P. Shi, " $H_{\infty}$ model reduction of Takagi-Sugeno fuzzy stochastic systems," IEEE Transactions on Systems, Man, and Cybernetics, Part B, vol. 42, no. 6, pp. 1574-1585, 2012.

[31] W. Yang and M. Liu, " $H_{\infty}$ filtering for nonlinear stochastic systems with sensor saturation, quantization and random packet losses," Signal Processing, vol. 92, no. 6, pp. 1387-1396, 2012.

[32] S. Xu, J. Lam, and T. Chen, "Robust $H_{\infty}$ control for uncertain discrete stochastic time-delay systems," Systems \& Control Letters, vol. 51, no. 3-4, pp. 203-215, 2004.

[33] P. Seiler and R. Sengupta, "An $H_{\infty}$ approach to networked control," IEEE Transactions on Automatic Control, vol. 50, no. 3, pp. 356-364, 2005.

[34] S. Sastry, Nonlinear Systems, vol. 10 of Interdisciplinary Applied Mathematics, Springer, New York, NY, USA, 1999.

[35] B. Zhang, S. Xu, and Y. Zou, "Improved stability criterion and its applications in delayed controller design for discrete-time systems," Automatica, vol. 44, no. 11, pp. 2963-2967, 2008.

[36] Y. He, G. P. Liu, D. Rees, and M. Wu, " $H_{\infty}$ filtering for discretetime systems with time-varying delay," Signal Processing, vol. 89, no. 3, pp. 275-282, 2009. 


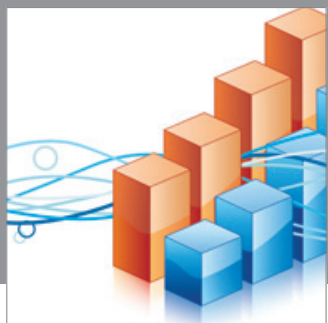

Advances in

Operations Research

mansans

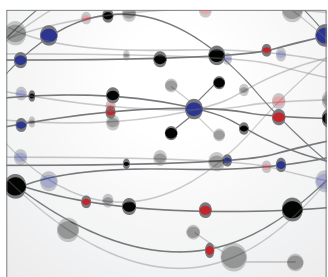

The Scientific World Journal
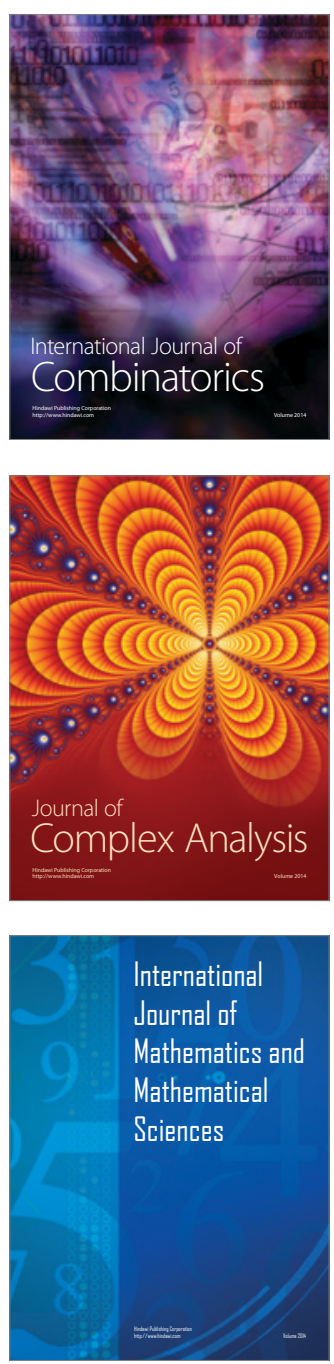
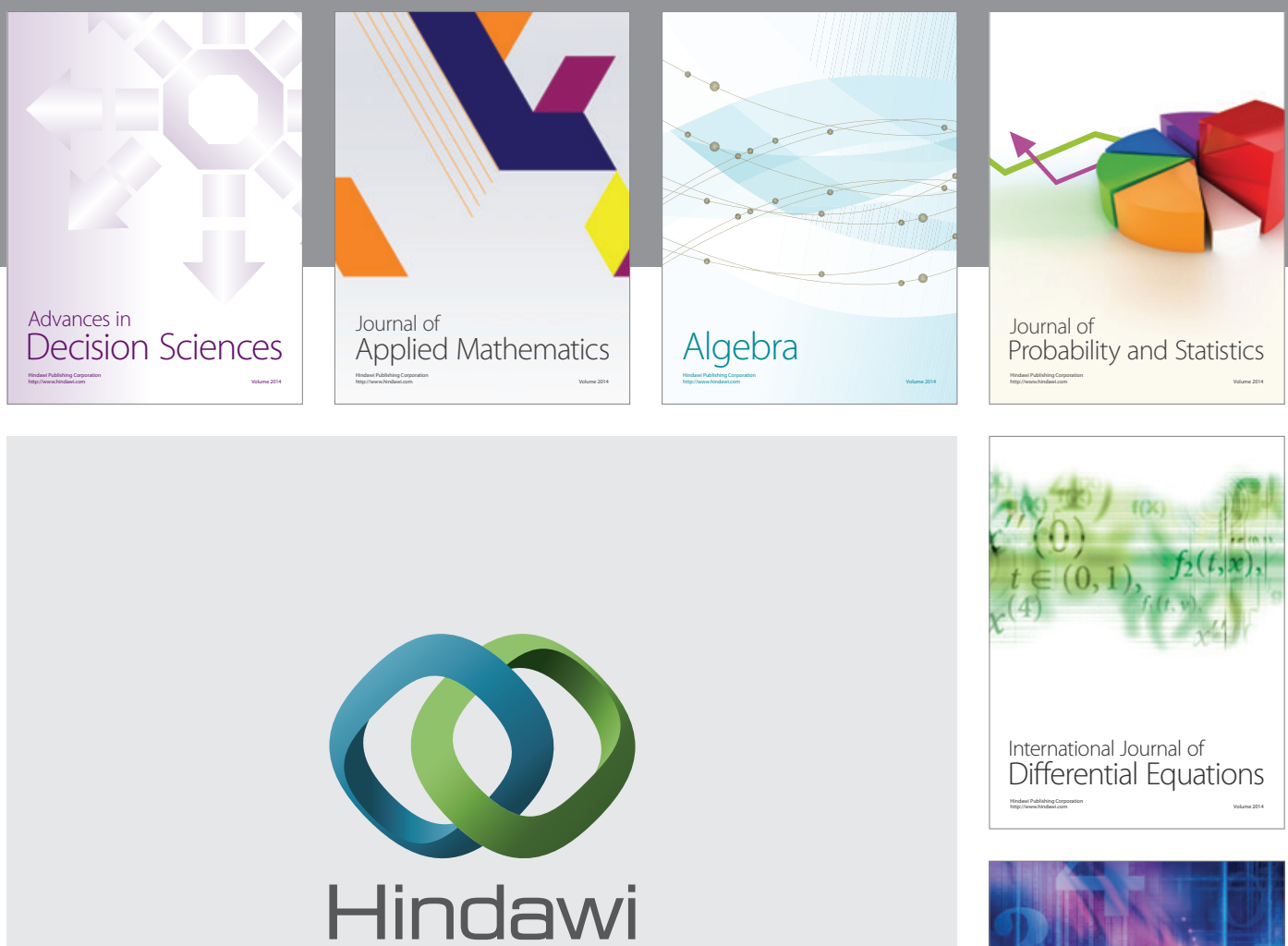

Submit your manuscripts at http://www.hindawi.com
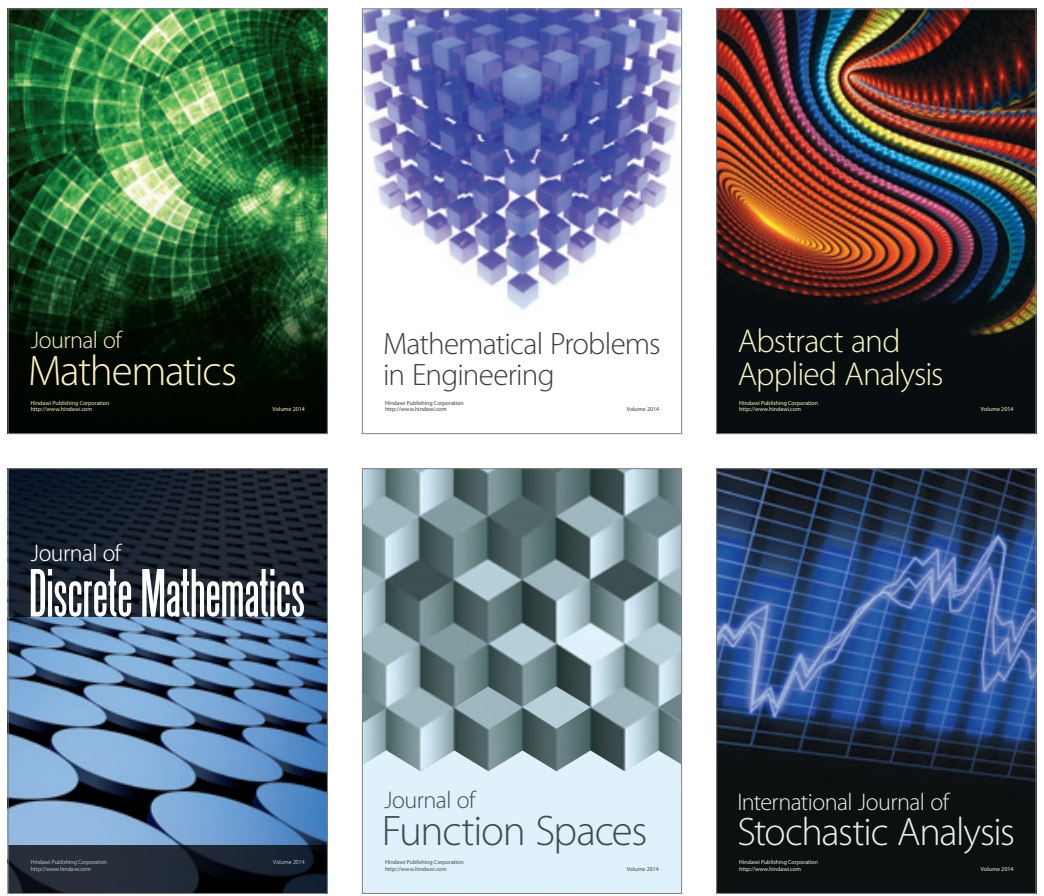

Journal of

Function Spaces

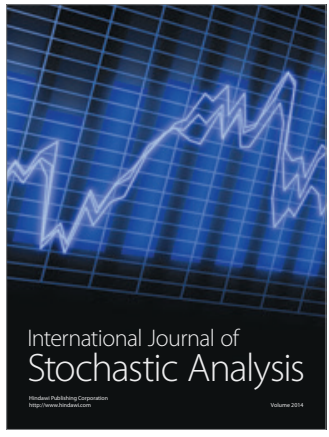

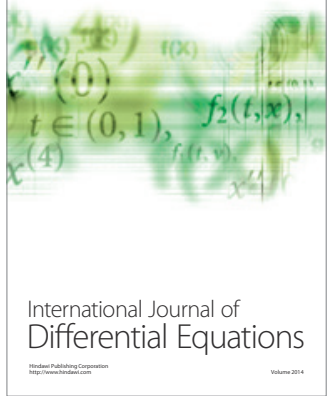
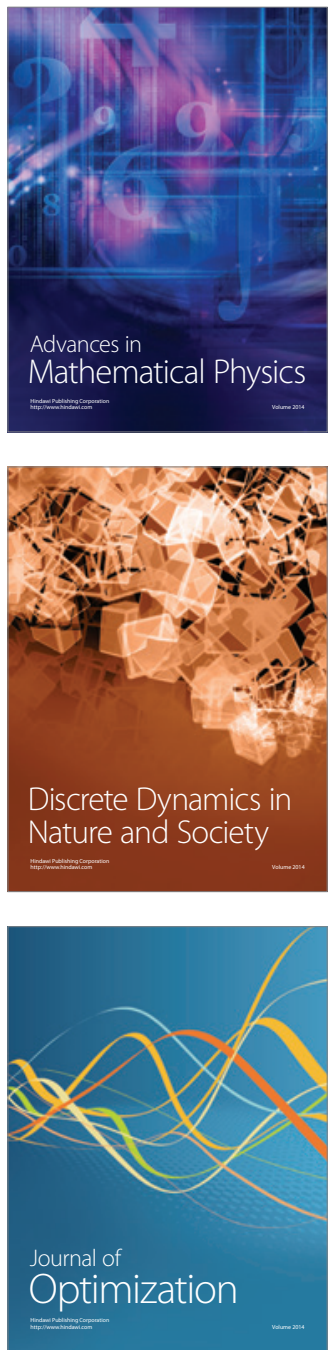